Each represents an extreme development in a particular direction, perhaps something of a caricature, but taken as a group they mark out the limits within which the future is likely to lie. The real future will therefore probably lie between these extremes, having some characteristics of each

The large differences between these four scenarios, all of which are plausible, may be shown by one example, the level of drug use in these alternative futures. The figure shows drug use expressed in terms of volume and monetary value compared with that in 1990. The estimated volume of drug use in the year 2005 ranges from 54 (risk avoidance) to 239 (free market unfettered), while the monetary value of drug use ranges from 100 (risk avoidance) to 418 (technology on demand).

The scenarios are intended to confront key players in the drug business with possibilities that they have not foreseen. Policy makers may find it useful for showing the limits within which they can influence change and the factors which have to be taken into account. They will be able to consider the future role of the various key players-including prescribers, pharmaceutical researchers, and suppliers of over the counter medi- cines-against these various alternative backgrounds. Used methodically and creatively, scenario analysis can be a teaching device and a powerful technique for adjusting preconceptions in laying a basis for community policies well into the 21 st century.

1 Dukes MNG. Drug policies: the need for constructive criticism. Postmarketing Surveillance 1991;5:231-5.

2 Grabowski H. Pharmaceutical research and development: returns and risk. CMR Annual Lecture, 1991. Carshalton: Centre for Medicines Research, 1991.

3 Gilley J. Towards rational prescribing. $B M 7$ 994;308: 731-2.

4 Ray AW, Griffin MR, Avorn J. Evaluating drugs after their approval for clinical use. NEngl f Med 1993;329:2029-32.

5 Dukes MNG, ed. Drug utilisation studies. Methods and uses. Geneva: WHO Dukes MNG, ed. Drug utilisation studies. Methods and
Regional Publications, 1993. (European Series No 45.)

6 Taylor D. Prescribing in Europe-forces for change. BMF 1992;304: 239-42.

7 Young FE. Ethical and financial considerations in 3rd-party support of investigational cancer therapies. Cancer 1993;72 (9 suppl):2854-8.

8 Griffin MT. AIDS drugs and the pharmaceutical industry: a need to reform Am ₹ Law Med 1991;17:363-410.

9 Leufkens HG, Urquhart J. Prescriber profile and postmarketing surveillance [letter]. Lancet 1993;342:1178.

10 Folb PI. Scenario analysis of the future of medicines. Lancet 1993;342:294

11 Wack P. Scenarios: uncharted waters ahead (part I). Harvard Business Review 1985;Sept-Oct:73-89.

12 Wack P. Scenarios: shooting the rapids (part II). Harvard Business Review 1985; Nov-Dec:139-50.

13 Schwartz $P$. The att of the long view. Planning for the future in an uncertain world. New York: Bantam Books, 1991.

(Accepted 12 October 1994)

\title{
The profession of medicine
}

\author{
Kenneth Calman
}

\section{Where there is no vision the people perish-PROVERBS}

It seems timely to define the purpose of medicine and examine the concept of a profession. This paper does so in the wider context of health, values in society, and the need to involve patients and the public as a whole. The author looks closely at what doctors do and concludes that making the diagnosis is a key element. The consultation is the building block for resource allocation. In addition to the diagnosis it sets out the prognosis and possible treatment and emphasises the importance of communicating these to the patient. Looking at the kind of doctor we need raises such issues as ethical standards, continuing professional development, team working, clinical standards, quality, outcomes, and research and development. Throughout, the role of education is seen as crucial. Leadership and vision are required by senior members of the profession if the opportunities presented are to be developed further.

There is increasing public and professional interest in medicine, with questioning of professional standards and the quality of care. Public expectations of the level of service to be delivered are rising. It is timely, therefore, to review the role and purpose of medicine and the concept of a profession.

At the outset let me make it clear that the medical profession in Britain provides a service to individuals and the population which is the envy of the world. The commitment to provide such a service within the NHS is real and deeply held. Because of this the profession has nothing to fear by being open and encouraging debate on how services could be even better. And that is the thread which goes through this paper-that doctors provide one of the most valued services in Britain and are held in high esteem but will continue to do so only if they take the lead in promoting change and improvement. In other words doctors must consider their role as a profession.
Before getting into the substance of the paper let me first try to put this topic into context and set out six key issues which govern my thinking on health and health care. I have developed these elsewhere, and they include the importance of health, of patient and public involvement, in measuring outcomes, intelligence systems, ethical issues, and the education and research base. ${ }^{1}$

This paper will consider some of these issues in more detail.

\section{What is the purpose of medicine?}

The purpose of medicine is to serve the community by continually improving health, health care, and quality of life for the individual and the population by health promotion, prevention of illness, treatment and care, and the effective use of resources, all within the context of a team approach. This emphasises a series of key issues, including the focus on patients, health and quality of life, the use of resources, and the team approach. Each of of these I will discuss in more detail below.

It was Disraeli who said, "The health of the people is really the foundation upon which all their powers of state depend." This emphasises the importance of the health of the nation for economic and social wellbeing of the population, in addition to its importance to the individual.

\section{What is a profession?}

It is not easy to define a profession, but it is likely to have some or all of the following characteristics. It is a vocation or calling and implies service to others; it has a distinctive knowledge base which is kept up to date; it determines its own standards and sets its own examinations; it has a special relationship with those whom it serves-patients, clients; it has particular ethical principles-the ethical base; it is self regulating and is accountable to patients and to the profession itself. 
Each of these characteristics is important in ensuring public confidence in medical practice and in retaining credibility. From each of these can be derived further, or secondary, principles and values in medicine, such as the importance of continuing education, quality, and the role of research and development. Changing the culture and ethos of the health service has sharpened the focus on the value base of medicine. These principles, or similar ones, are crucial and are not dependent on the organisation and structure of the health service and require wide debate.

\section{What do doctors do?}

What do doctors do? This is broadly about the role of medicine, though this question can also be viewed in a more restricted way to refer to the particular contribution of clinical practice. We should also ask, "what do doctors do that others don't?" Clearly, doctors see patients, do investigations, prescribe or carry out treatment, do research, and teach. They care for patients, and this aspect-which includes commitment and compassion-is very important. We must not undervalue this aspect of a doctor's work and the skills required to do it well. Some doctors cover whole families, or whole communities, and bring to them the same kinds of skill and expertise. But it could be said that other professional groups do many of the same things. Doctors have no monopoly of caring, investigations, research, or even treatment, though there remain many forms of treatment which are carried out only by doctors.

However, there is one aspect of practice of profound importance which is generally carried out by doctorsthat is, in making a diagnosis and assessing its consequences. The implications are crucial. Firstly, the consultation, the accumulation of information, the making of a diagnosis, and setting out the prognosis and possible treatment make up the basic building blocks of health care and resource allocation. Further, a key part is the communication of the diagnosis, prognosis, and treatment to the patient, or to the community. Making a diagnosis is not simple and straightforward even when the name of the "disease" is well recognised. Social and family implications are crucial, emphasising the holistic nature of the concept and of patient care. It is a person with an illness, not just a label, and the wider implications of the "diagnosis" need to be emphasised. The process of making a diagnosis through history taking, physical examination, and investigations is also crucial and cannot be fragmented by detaching parts in the systematic search for a diagnosis. At this stage others may take the lead role in following through treatment, counselling, etc, but the fundamental issue remains the diagnosis, its communication, and the setting out of a plan of action, agreed with the patient or the community, on the management of the problem.

This raises a further issue of what is meant by a diagnosis and what are its dimensions. This can clearly range from a provisional working hypothesis through to histopathological confirmation. In each instance there are likely to be uncertainties, and one of the skills of the craft of medicine is to be able to communicate such uncertainties. Learning to live with uncertainty as a patient is not easy and, as the progress of the disease process evolves, continuing support and explanation are required. However this emphasises again the central nature of the diagnosis, its consequences in health care, and the need for a holistic approach.

In most instances it is the diagnosis which will determine the resource required (in some instances the patient will quite properly not wish for the treatment) and it therefore follows that it is the technology which will further define that resource. This may be surgical
Box 1-The patient perspective in clinical practice

- What is wrong with me?-The diagnosis

- What does this mean for me?-The prognosis

- What can be done for me?-The caring and management component

- What can I learn from this patient?-The research dimension

- What can others benefit?-The public health dimension

- What can I teach others from this experience?-The educational opportunities both for patients and for professionals

treatment, a medicine, physiotherapy, a special diet, counselling, etc-the range is enormous. As the technology changes so the resource will vary, and the rate of change in clinical practice is increasing every year. This means that doctors must keep up to date and be sure that new treatments are worth while before they are widely introduced, emphasising the role of research and development, education, and of outcome assessment. These issues will be discussed further below.

Doctors also have responsibilities for the use of resources. This should not be narrowly defined in terms of funding, though clearly this is central. It should also include the appropriate use of time, skills, and the facilities available. A doctor who spends two hours with one patient cannot spend these hours with others. Similarly, if a sum of money is spent on one patient it will not be available for another. Resources are and always have been finite. The classic dilemma of the doctor is how to do the best for one patient without disadvantaging another. Trust is a key element in this process. The fact that this is difficult must not mean that doctors abrogate their responsibilities. Key to this is an understanding of the outcome of the process of care and the ability to make such difficult judgments on the basis of knowledge and evidence. It may be necessary to separate decision making at the bedside from resource allocation at a higher level.

Thus doctors could be seen to have three broad roles-firstly, to provide high quality care, and in particular to be concerned with diagnosis, prognosis, treatment, and the planning of care and the communication of this to the patient; secondly, to be concerned with the individual and with the community; thirdly, to manage resources effectively, including skills, time, facilities, and finance. This can be combined with a view from the perspective of the patient to give a different picture. The purpose of the doctors can then be redefined as answering a series of questions posed by the patient (box 1). ${ }^{2}$

\section{What kind of doctor do we need?}

If we agree about the purpose of medicine, what a profession is, and what doctors do it becomes possible to consider the kind of doctor we need. This is far from easy, as a consideration of a range of specialties identifies several characteristics. Is it possible, therefore, to describe a core of characteristics for all doctors? The following might be included and can best be classified as key values expected of all doctors, which would be independent of the specialty and indeed independent of the structure and organisation of the health service.

\section{KEY "VALUES" EXPECTED OF DOCTORS}

High standard of ethics-From the time of the Hippocratic oath medical practice has always had a very strong ethical foundation, and rightly so, as it is 
one of the key features of a profession. ${ }^{3}$ More than ever the ethical principles associated with clinical practice need to be debated and clarified. New procedures and ethical dilemmas arise constantly. Greater public awareness of the issues means that the debates are no longer confined to professional audiences and it is appropriate that the wider public is included. There is greater scrutiny of professional practice, and standards are now openly discussed in the media and public fora. The profession has nothing to fear from such debates as long as it is not defensive or secretive.

Continuing professional development is an issue which is broader than continuing education, which is discussed below. It is concerned with personal growth and satisfaction with professional work. It is an issue which has been neglected in recent years but, with the changing role of the consultant, it is one which will need to be looked at afresh in the near future.

The ability to work in a team-As medicine and health care increase in complexity so it becomes even more necessary to be sure that all the skills of professions other than medicine are utilised to the full. This means working and learning in teams, but it does not mean relinquishing the key patient-doctor relationship, which is so central to the therapeutic process. ${ }^{4}$

Concern with health as well as illness is a key issue. Are doctors to be concerned only with those who are ill or do they have a wider role in the community?

Patient and public focused-The purpose of medicine, if you agree with the definition given above, is precisely to do with serving that patient and the public. Perhaps we should be more willing to say this explicitly rather than implicitly. There is great energy and power in this process if it can be harnessed effectively.

Concern with clinical standards, outcomes, effectiveness, and audit-More and more of the care given to patients and the treatment offered will be based on proper outcome based evidence. This is not to deny innovation or to stifle research and development. Rather the opposite. Standards record where we are now; research and innovation should take us to new levels of quality and care. It is clear from many studies that there are variations in treatment and outcomes across Britain. Some of these are understandable and explainable. Others are not. It is this aspect which from a public point of view requires resolution. Audit is a tool which has value in measuring that quality of care provided. It is only one tool but an important one to assure quality. As part of professional practice all doctors should be involved in auditing clinical work.

Ability to define outcomes-Outcomes in some instances are not easy to define but are an important professional challenge. The use of guidelines, which can be seen as no more than the formalisation and clarification of good clinical practice, is part of the process of care and is not an end in itself. Guidelines

\section{Box 2-Measuring the quality of a clinical unit}

- Clinical records system in place which documents relevant information

- A note of the major procedures, diseases, etc which are seen in the unit

- Guidelines or standards for each of these major topics, clearly documented

- Audit procedures in place to monitor the performance in each area

- Documentation of all new procedures, with evidence of effectiveness and an evaluation of the outcome

- Evidence of patient or public involvement in these guidelines-for example, from the preparation of patient information leaflets to patient participation groups

- Staff appraisal procedures in place to provide feedback on performance

- Documentation of educational programmes available to all staff

- Teamwork in evidence, utilising the skills of all staff

- Management of resources as evidenced by an awareness of the consequences of mismanagement. This should include time, skills, facilities, and finance

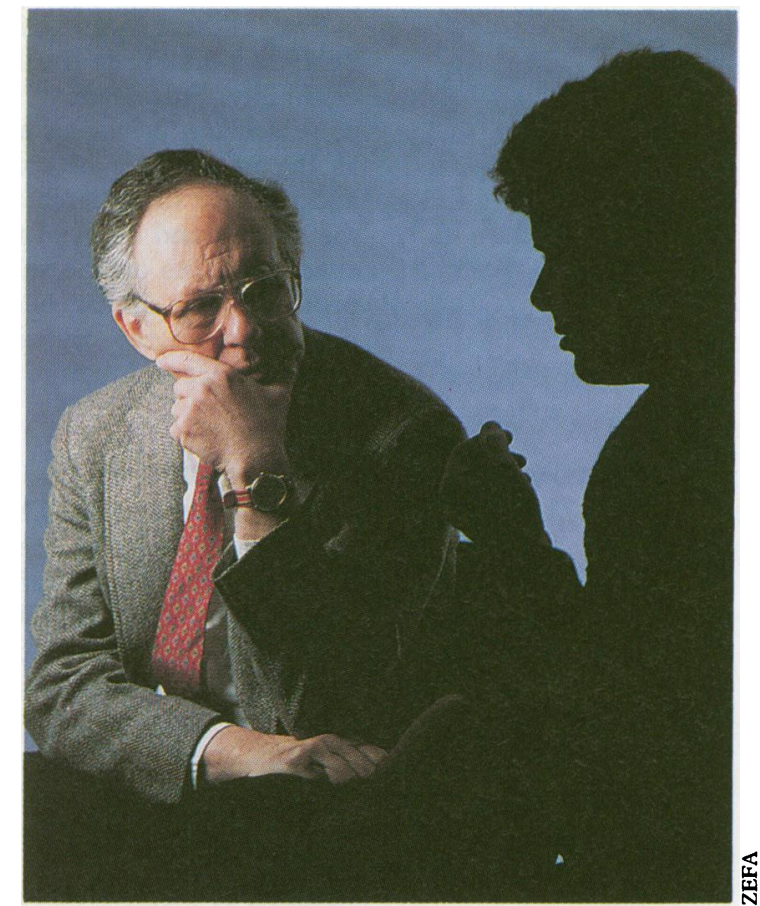

Practising medicine is a privilege, and doctors must be accountable

need to be feasible, to encourage local involvement, and not to inhibit new methods of management. Rather they should be a base from which to build. They should help us to understand and explain variations in care.

Interest in change and improvement, research, and development-Medicine cannot and should not stand still. It is continually evolving and improving. All doctors need to be involved in changing and improving clinical practice. Indeed they have an obligation to be so, though this does not mean that all need to be involved in "research." This key value, however, emphasises the importance of academic input and of teaching.

Ability to communicate - Of all complaints against doctors, problems of communication must be one of the greatest. Yet it is perhaps the key part of the function of the doctor if you accept that making a diagnosis, assessing prognosis, and defining treatment are central roles for the doctor. Arrogance and discourtesy reflect badly on a profession whose primary purpose is to care for patients. The importance of communication (a two way process) is increasingly being recognised in medical schools, and role models (consultants and general practitioners) must also recognise the effect of the "hidden agenda" (their attitudes and behaviour) on medical students and postgraduates.

\section{What are the implications of these questions?}

Some of the implications of these questions have been discussed. Others will be picked up now.

Medical education: the knowledge base and the curriculum-From my own point of view medical education is the most important issue. We must see medical education as a continuum, including continuing medical education, and recognise that doctors have an ethical obligation to keep up to date. The knowledge base is part of being a professional and it should be being continually updated and replenished. There is much to be said about education and training, but in this paper only one further point will be made. This is to distinguish between the two concepts. To be trained is to have arrived; to be educated is to continue to travel. Doctors need to have a broad vision of the world and be able to change and adapt as the knowledge base 
changes. They need to have outside interests and be rounded people, with breadth as well as depth.

Public involvement is easy to say but difficult to carry out. Who are the public and how can we best achieve their input? Perhaps the best solution is to be much more specific and to select the public or patient group to fit the issue. I am convinced, however, of the value of involvement and of ensuring that the patient dimension is heard and listened to.

Quality is difficult to define both at an individual practitioner level and at the level of a clinical unit. A question which is commonly asked relates to how it might be possible to measure quality in a clinical unit. Box 2 gives some ideas.

Theoretical base-Models of health and disease are required to provide a foundation for progress. The development of general theories of health and illness would help to underpin the understanding of disease and the maintenance of health. ${ }^{5}$

Doctors in management is a major issue at present and one with which the profession must come to terms. Do doctors wish to be involved in management, and if so at what level? Clearly they should be involved in managing patients, their own time, and the resources at their disposal. But what of the tasks beyond that, as clinical directors, medical directors, chief executives, general managers? Not all doctors will wish to be involved at all levels, but the profession must consider carefully the opportunities, be able to take them, and support those who do.

Leadership and vision are certainly important attributes and more than ever we need them in the senior members of the profession. We must be able to look beyond the present and to identify where we would like to be and the kind of values which are necessary. The National Commission on Education stated, "education is the process by which values are transmitted from one generation to another." We must be sure what they are and develop strategies to get there. The role of the teacher is crucial.

Professional organisation and self regulation-One issue which is increasingly surfacing is the organisation of the profession and its need to remain self regulating. Under increasing scrutiny the profession will need to look carefully at itself and ensure that from a public and professional point of view all steps are taken to assure quality and that "self" regulation means just that. Unless the profession takes care of its problems others will do it for us. Practising medicine is a privilege and we need accountability for it.

\section{Public health implications}

- The importance of communication and involvement of patients and the public needs to be emphasised

- Professional values, the ethical base, education, research, and standards provide the basis of quality assurance in clinical practice

- Using resources effectively requires an understanding of the diagnosis, prognosis, and the outcome of the process of care

- Doctors have a responsibility for health, as well as illness, and for the community they serve

Cooperation among specialties-With increasing specialisation there has been a tendency recently for the profession to speak with different voices and to appear fragmented. Perhaps the time has come to be more integrated and collegiate. For many years there has been discussion about the establishment of an academy or institute of medicine in Britain, perhaps with regional chapters. Maybe this is an idea worth reviving. Certainly there is a need for greater cooperation between branches of the profession, and in particular I would single out the importance of links between primary and secondary care.

\section{What action is required now?}

I believe that what we need is a full debate on the purpose of medicine and its basic values; a continuing review of medical education in a time of change-this is a key issue; an examination of standards and quality in clinical practice; further consideration of the organisation of medicine, looking to the year 2000 and beyond; and a recognition of the responsibility of the profession to take these forward.

1 On the state of the public health. London: HMSO, 1992.

2 Calman KC. Medicine in the 1990s. Ann Acad Med Singapore 1991;20:287-94. 3 Calman KC. Do we need a new Hippocratic Oath? Liverpool Medical Transactions 1992-3:29-33.

4 Calman KC. Working together, teamwork. Fournal of Interprofessional Care 1994:8:95-9.

5 Calman KC. The future of the nation's health. Update 1992;April 15:709-23.

6 National Commission on Education. Learming to succeed. London: Heinemann 1993.

(Accepted 12 October 1994)

\section{ANY QUESTIONS}

Has any work been done on biological washing powders as a new allergen? It is increasingly difficult to buy non-biological washing powders.

Detergent products containing proteolytic enzymes derived from Bacillus subtilis were introduced in the 1960s. Reports of allergic reactions among workers in the industry appeared soon afterwards. In 1969 Flindt published an account of reactions, including late onset asthma, after exposure to $B$ subtilis detergent dust. ${ }^{1}$ Those affected were workers in factories producing the washing powder. They were shown to have immediate reactions on skin prick testing with the enzyme preparation from $B$ subtilis. These reports and worries about sensitisation of consumers led to closer environmental control in the industry and a change in the product to encapsulate the enzyme or to produce a particulate product.

There was considerable anxiety over the risk to consumers using the biological powders, with reports of symptoms and positive reactions on skin prick testing. ${ }^{2}$ The change in the manufacturing process seems to have removed the problems of sensitisation among consumers, ${ }^{3}$ although people with asthma might conceivably be affected.

Problems can still arise in the industry, particularly when there are problems in the manufacturing process. ${ }^{4}$ P J REES, consultant physician, London

1 Flindt MLH. Pulmonary disease due to inhalation of derivatives of Bacillus subtilis containing proteolytic enzyme. Lancet 1969;i:1177-81.

2 Pepys J, Wells IC, D'Douza MF, Greenberg M. Clinical and immunological responses to enzymes of Bacillus subtilis in factory workers and
a logical responses to enzymes of Bacil

3 Pepys J, Mitchell J, Hawkins R, Malo JL, Ashforth GK, Wilson ER. A longitudinal study of possible allergy to enzyme detergents. Clin Allergy 1985;15:101-6.

4 Perdu D, Lavaud C, Cossart C, Legrele S, Passemard F, Deltour G, et al. Detergent enzymes: has the risk of occupational sensitization disappeared? Rev Mal Respir 1992;9:443-8. 